\title{
frottement latéral des pieux dans les sables carbonatés
}

\author{
skin friction of piles in calcareous sands
}

\author{
P. LE TIRANT - J.-F. NAUROY - F. BRUCY \\ Institut Français du Pétrole* \\ H. BARTHÉLÉMY \\ Solmarine ${ }^{*}$ \\ J.-P. KERVADEC \\ Total-CFP $\cdots$
}

Rev. Franç. Géotech. n 49, pp. 51-66 (octobre 1989)

\section{Résumé}

Les formations carbonatées rencontrées en de nombreuses zones d'activités pétrolières en mer posent de difficiles problèmes de dimensionnement et de mise en place des pieux de fondation ou d'ancrage des ouvrages de production. Depuis 1978, I'ARGEMA, Solmarine et Total-CFP ont entrepris un important programme de recherche sur le comportement des pieux battus, forés et cimentés ou battus-cimentés dans les sables carbonatés. Les expérimentations conduites sur le site de Plouasne avec des pieux d'une longueur de $20 \mathrm{~m}$ ont largement contribué à l'amélioration du choix et du dimensionnement des pieux dans les formations carbonatées en mer.

\footnotetext{
Abstract

The calcareous formations encountered in many zones of offshore petroleum activity raise difficult problems concerning the design and installations of foundation and anchoring piles for production platforms. Since 1978, ARGEMA, Solmarine and Total have undertaken an important research program on the behaviour of piles driven, drilled and grouted or driven and grouted in calcareous sands. Experimentation carried out at in Plouasne site with piles about twenty meters long, have gone a long way towards improving the choice and design of piles in offshore calcareous formations.
}

\footnotetext{
- 1 et 4, avenue du Bois-Préau, 92506 Rueil-Malmaison.

$\because 6$, rue de Walford, 92000 Nanterre.

*.. Cedex 47, 92069 Paris La Défense.
} 


\section{INTRODUCTION}

Les formations carbonatées rencontrées en de nombreuses zones d'activités pétrolières en mer posent de difficiles problèmes de dimensionnement et de mise en place des pieux de fondation ou d'ancrage des ouvrages de production. Le frottement latéral des pieux battus - procédé habituel de mise en place des pieux en mer - est généralement faible, voire négligeable dans les formations carbonatées compressibles. Cette situation a conduit les opérationnels à rechercher d'autres solutions pour la réalisation des fondations marines soit par forage et cimentation des pieux tubulaires, soit plus récemment par injection de ciment le long des pieux tubulaires battus. Dans ce contexte l'ARGEMA, Solmarine et Total-CFP ont effectué depuis plusieurs années un important programme de recherche sur le comportement et le dimensionnement de différents types de pieux dans les sables carbonatés, compressibles.

Cette communication décrit brièvernent les différentes phases de l'expérimentation de pieux conduite, de 1982 à 1986, sur le site de sable carbonaté (falun) très compressible de Plouasne, à l'ouest de Rennes, pour la détermination du frottement latéral mobilisé par les divers types de pieux:

- pieu battu A de $30 \mathrm{~cm}$ de diamètre et de $23 \mathrm{~m}$ de long,

- pieu foré et cimenté $\mathrm{B}$ de $35 \mathrm{~cm}$ de diamètre et de $15 \mathrm{~m}$ de long,

- pieu battu et «réparé» par injection de ciment $\left(\mathrm{A}^{\prime}\right)$ depuis un forage extérieur au pieu.

- pieu battu et cimenté $C$ de $76 \mathrm{~cm}$ de diamètre et de $24 \mathrm{~m}$ de long, opéré par Solmarine. L'injection de ciment est effectuée au moyen de clapets disposés le long du pieu avant le battage (brevet Solmarine-Solétanche).

Tous les pieux étaient instrumentés sur toute leur longueur par trois séries de jauges de déformation disposées à $120^{\circ}$. Des capteurs de force et de déplacement en tête complétaient l'instrumentation.

L'expérimentation a comporté essentiellement des essais statiques en traction permettant d'obtenir la répartition du frottement latéral mobilisé par les différents types de pieux.

\section{SITE DE PLOUASNE}

Le choix d'un site expérimental de sable carbonaté compressible, s'apparentant à ceux rencontrés dans plusieurs zones en mer, a conduit pour l'expérimentation de pieux de fondations marines à identifier les formations de faluns du bassin de Rennes. Les diverses informations géologiques et géotechniques recueillies ont conduit, après une reconnaissance approfondie, à retenir le site de Plouasne situé à $40 \mathrm{~km}$ environ au nord-ouest de Rennes.

La figure 1 représente la coupe lithologique du sol de Plouasne. Après cinq mètres de sable silteux siliceux, le sol est constitué d'alternances de sables carbonatés grossiers et fins avec une proportion variable de fines et quelques couches très faiblement cimentées et peu épaisses. Les sables carbonatés appartiennent à une formation géologique d'áge Miocène, formée de débris de coquilles, d'algues et de coraux.

Les caractéristiques des sables carbonatés sont résumées sur le tableau 1.

Le niveau de la nappe phréatique se situe entre 2 et $2.5 \mathrm{~m}$ suivant les saisons: ainsi, la formation carbonatée située au-dessous de $5 \mathrm{~m}$ est en permanence saturée.

\section{PIEU A BATTU}

\subsection{Description du pieu A}

Le pieu $A$ battu sur le site de Plouasne est un tube ouvert de dimensions suivantes:

- diamètre extérieur : $298.5 \mathrm{~mm}$;

- épaisseur : $8 \mathrm{~mm}$ :

- longueur : $23 \mathrm{~m}$.

Le pieu a été instrumenté par des jauges de déformation collées sur la surface extérieure, selon trois génératrices à $120^{\circ}$ et réparties sur onze niveaux espacés de deux mètres (fig. 2).

Tableau 1 - Caractéristiques des sables carbonatés de Plouasne

\begin{tabular}{|c|c|c|c|c|c|c|c|}
\hline $\begin{array}{l}\mathrm{D}_{50} \\
(\mathrm{~mm})\end{array}$ & $\begin{array}{l}\mathrm{D}_{10} \\
(\mathrm{~mm})\end{array}$ & $\mathrm{CaCO}_{3}$ & $\begin{array}{l}\gamma \mathrm{d} \text { in situ } \\
\left(\mathrm{kN} / \mathrm{m}^{3}\right)\end{array}$ & $\begin{array}{c}\gamma \mathrm{d}_{\max } \\
\left(\mathrm{kN} / \mathrm{m}^{3}\right)\end{array}$ & $\begin{array}{c}\gamma d_{\min } \\
\left(\mathrm{kN} / \mathrm{m}^{3}\right)\end{array}$ & $\begin{array}{c}\text { Indice } \\
\text { de compressibilité } \\
\text { dans le domaine } \\
50 \text { à } 800 \mathrm{kPa} \\
\text { (Nauroy et al, 1988) }\end{array}$ & $\begin{array}{c}\text { Angle } \\
\text { de frottement } \\
\text { caractéristique } \\
\varphi_{\text {ca }}\left({ }^{\circ}\right)\end{array}$ \\
\hline 0,4 à 0,8 & $<0,04$ & 80 à 90 & 12 à 15 & 12,7 à 15 & 10,4 à 11,3 & 0,06 à 0,3 & 36 à $45^{\circ}$ \\
\hline
\end{tabular}




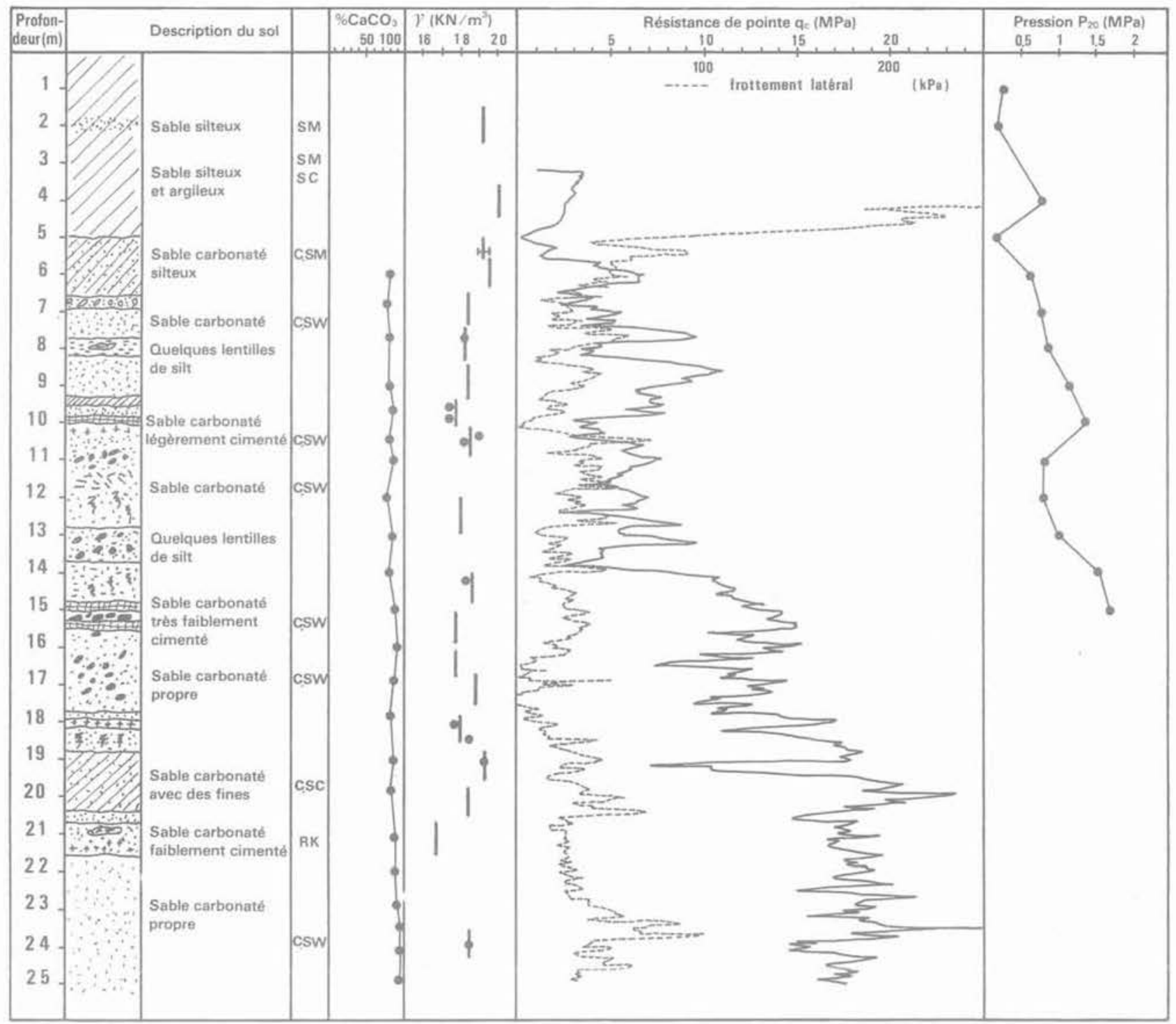

Fig. 1. - Description lithologique du site de Plouasne.

Fig. 1. - Lithological description of Plouasne site.

\subsection{Battage du pieu $A$}

Le pieu $A$ a été battu en juin 1982 avec un marteau Delmag D22 jusqu'à une profondeur de $22,5 \mathrm{~m}$. Les mesures dynamiques effectuées pendant le battage puis au cours de surbattages, avec une masse de $400 \mathrm{~kg}$, montrèrent que le frottement latéral dans les sables carbonatés était très faible (BRUCY et al., 1988).

\subsection{Essai de traction}

Un mois après l'installation par battage, le pieu a été soumis à un essai statique en traction, T1.

Pour cet essai, la charge était reprise par un bâti de réaction (fig. 3) et appliquée au moyen d'un vérin hydraulique. L'effort était mesuré par un capteur placé entre le pieu et le vérin.
Les mesures de déplacement de la tête du pieu étaient effectuées par des capteurs prenant appui sur deux bases fixes métalliques de six mètres de longueur chacune. Les appuis des bases fixes étaient situés à $3 \mathrm{~m}$ de l'axe du pieu.

Les bases fixes étaient protégées des dilatations thermiques par une enceinte en polystyrène.

Les différentes mesures de charge en tête, de déplacement et d'élongation des jauges étaient enregistrées par une chaîne d'acquisition Hewlett-Packard.

La charge limite n'a pas dépassé $75 \mathrm{kN}$. La couche de sable silteux de 0 à $4,2 \mathrm{~m}$ reprend les efforts les plus importants. La couche de sable carbonaté de 6,2 à $22,5 \mathrm{~m}$ ne reprend pratiquement pas d'effort. Le frottement latéral moyen dans cette formation est inférieur à $3 \mathrm{kPa}$. 

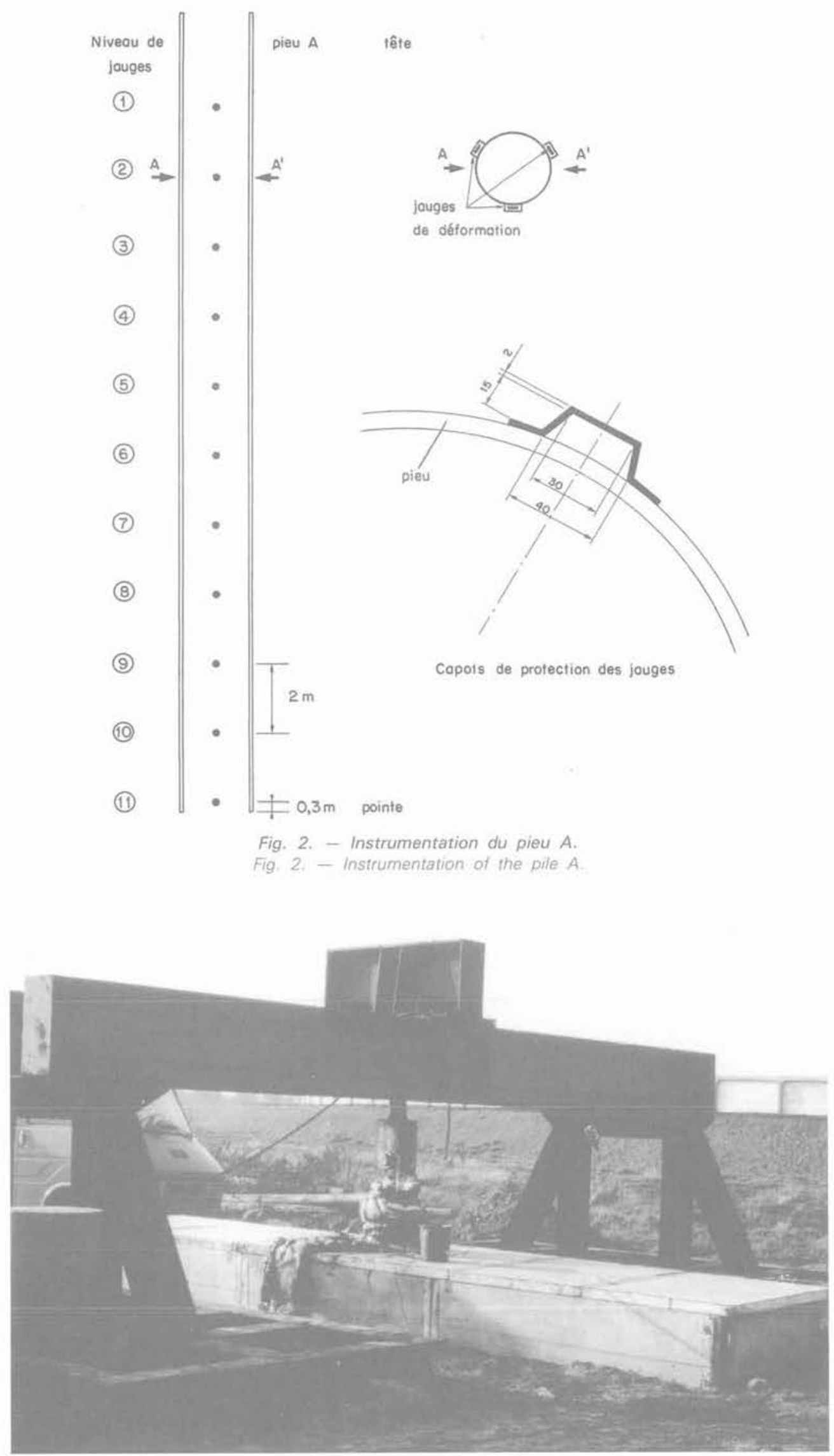

Fig. 3. - Le pieu A et le bâti de réaction.

Fig. 3. - The pile A and the reaction frame. 


\section{PIEU B FORÉ ET CIMENTÉ}

Le très faible frottement latéral mobilisé par le pieu A conduisait à la mise en place, sur le même site, d'un pieu foré et cimenté $B$ afin de vérifier les possibilités de ce type de fondation dans les formations carbonatées compressibles.

\subsection{Description du pieu $B$}

Le pieu $B$ est un tube de dimensions:

- diamètre extérieur : 219,1 mm;

- épaisseur : $10 \mathrm{~mm}$;

- longueur : $16 \mathrm{~m}$.

Le pieu a été instrumenté par des jauges de déformation collées sur la surface extérieure, selon trois génératrices à $120^{\circ}$ et réparties sur sept niveaux espacés de $1,75 \mathrm{~m}$ dans la partie destinée à être cimentée (fig. 4).

\subsection{Installation du pieu B}

La mise en place du pieu s'est effectuée en septembre 1983. Les huit premiers mètres de terrain ont été forés à l'eau avec un outil de $38 \mathrm{~cm}$ de diamètre. $\mathrm{Ce}$ trou fut ensuite tubé sur $7 \mathrm{~m}$. Le forage a été poursuivi, toujours à l'eau, avec un outil de $31 \mathrm{~cm}$ de diamètre jusqu'à la profondeur de $15,3 \mathrm{~m}$. Le trou fut alors nettoyé par «air lift». Après la descente du pieu dans le trou, l'annulaire entre le pieu et le terrain a été rempli avec un coulis de ciment sur une hauteur d'environ $7 \mathrm{~m} .590$ litres de coulis de ciment. préparé à un poids volumique de $19,5 \mathrm{kN} / \mathrm{m}^{3}$, furent injectés par gravité à partir de la base du pieu.

\subsection{Essais de traction}

Un mois après la cimentation, le pieu a été soumis à un essai de traction statique $\mathrm{S}_{1}$. L'appareillage utilisé était le même que celui employé précédemment pour le pieu A. La charge était appliquée par incréments de $50 \mathrm{kN}$ jusqu'à $1150 \mathrm{kN}$, maximum compatible avec l'équipement utilisé; la charge limite n'a pas été atteinte.

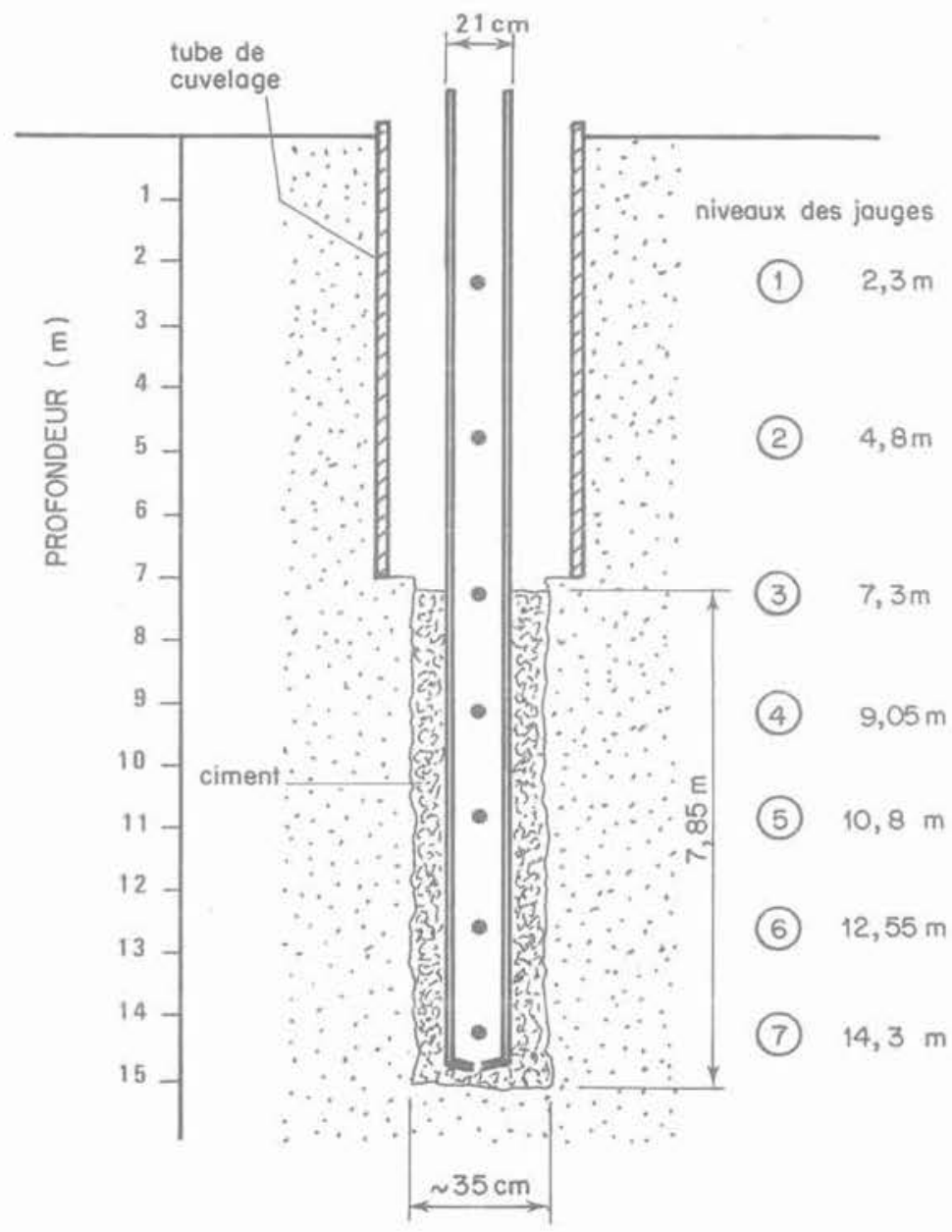

Fig. 4. - Le pieu B après cimentation. Fig. 4. - Pile B after grouting. 


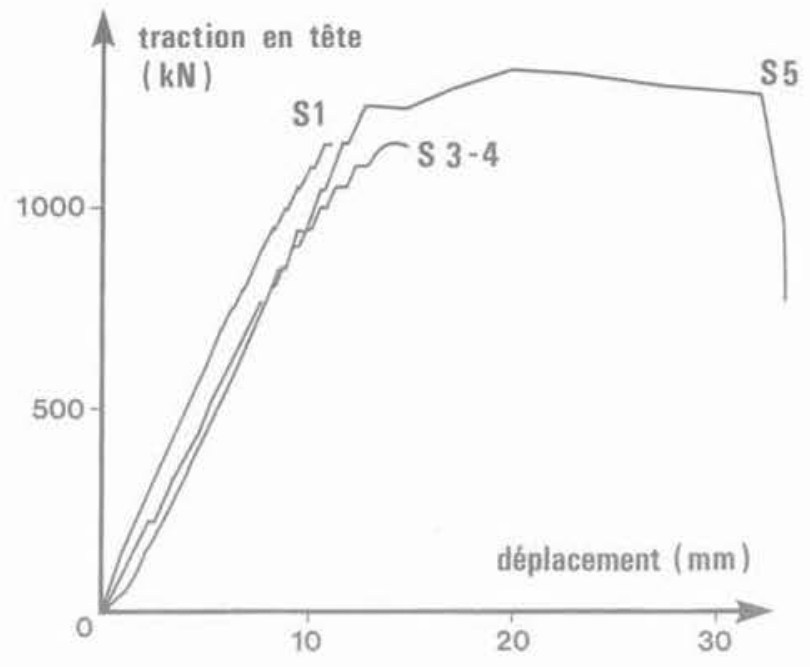

Fig. 5. - Résultats des essais de traction sur te pieu B. Fig. 5. - Results of tension tests on pile B

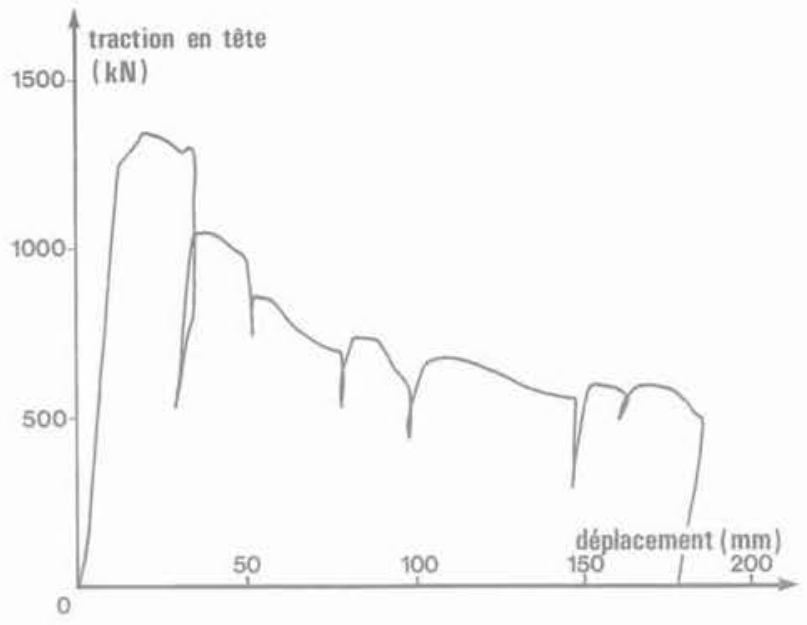

Fig. 6. - Capacité résiduelle du pieu B.

Fig. 6. - Residual capacity of the pile B.
Par la suite, en 1984, le pieu a été soumis a une série d'essais de chargements cycliques répétés en traction, la charge maximale ne dépassant pas $600 \mathrm{kN}$. A l'issue de ces essais cycliques, un essai de traction statique $\mathrm{S}_{34}$ fut conduit jusqu'à la charge maximale de $1150 \mathrm{kN}$, compatible avec l'équipement disponible, mais sans atteindre la rupture (NAUROY et al., 1985).

En 1986, profitant des moyens plus importants mis en place pour le pieu C (cf. chap: 5), un essai de traction statique $S_{5}$ fut conduit à la rupture (1 $340 \mathrm{kN}$ ) par incréments de $100 \mathrm{kN}$ (fig. 5). La charge fut ensuite diminuée puis remontée par paliers jusqu'à rupture et ainsi de suite pour un déplacement du pieu de $18 \mathrm{~cm}$ (fig. 6). La charge de rupture résiduelle se stabilise autour de $600 \mathrm{kN}$.

\subsection{Gaine de ciment autour du pieu B}

L'interprétation des mesures de traction sur le pieu $\mathrm{B}$ foré et cimenté nécessitait une connaissance précise de la géométrie de la gaine de ciment. Après découpage du sol autour du pieu par vibrofonçage d'une palplanche, le pieu a été extrait du sol sans effort notable. L'examen attentif du pieu a montré que le diamètre extérieur moyen de la gaine de ciment était d'environ $35,3 \mathrm{~cm}$ sur une longueur de $7 \mathrm{~m}$ (fig. 7). On a pu observer un léger décentrement du tube dans le trou de forage durant la cimentation.

\subsection{Frottement latéral mobilisé}

La figure 8 montre les courbes de mobilisation du frottement latéral par niveau. Le frottement est assez uniforme sur toute la hauteur cimentée. Le frottement maximal moyen est de l'ordre de $170 \mathrm{kPa}$ mobilisé

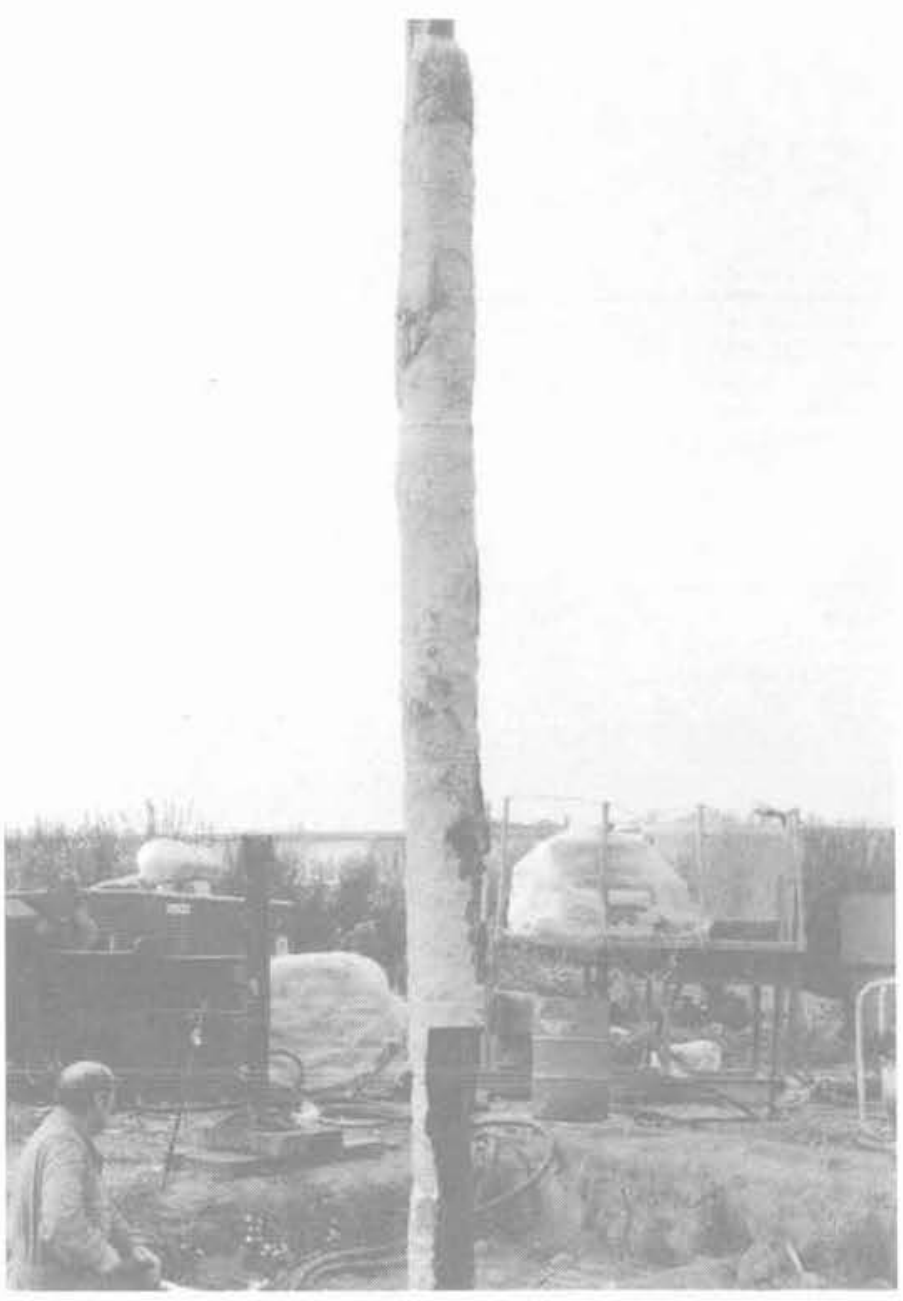

Fig. 7. - Gaine de ciment autour du pieu B. Fig. 7 - Grout sheath around the pile B. 


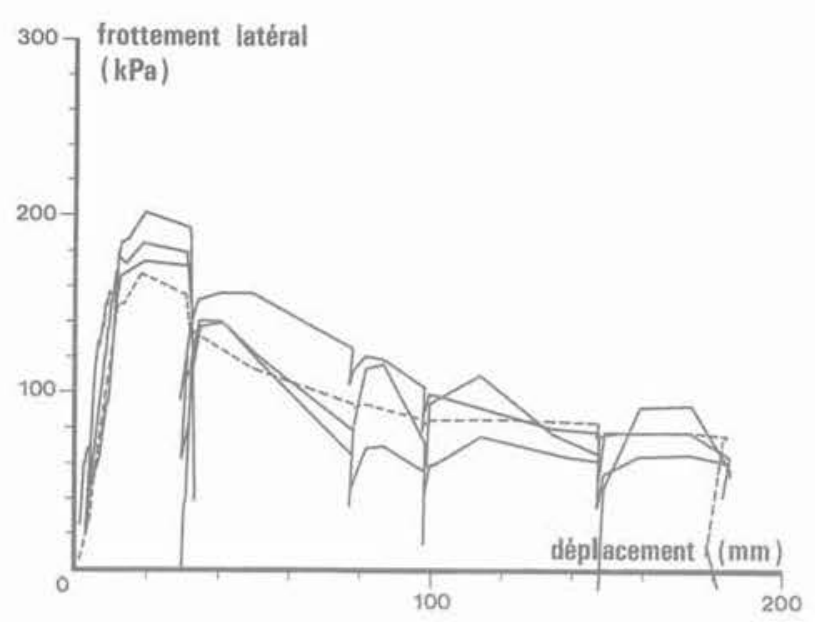

Fig. 8. - Frottement latéral mobilisé par le pieu B. Fig. 8. - Skin friction mobilized by the pile $B$.

pour un déplacement de 3 à $5 \mathrm{~mm}$. Le frottement décroît ensuite avec le déplacement du pieu et tend vers une valeur résiduelle de lordre de $70 \mathrm{kPa}$.

\section{PIEU A' BATTU ET RÉPARÉ PAR INJECTION}

Les résultats encourageants obtenus avec le pieu foré et cimenté $\mathrm{B}$ conduisaient à rechercher des solutions alternatives plus économiques par l'association du battage et de la cimentation. Dans cette optique, et dans la perspective de réparations de fondations d'ouvrages réels existants, une tentative de réparation du pieu battu A fut réalisée en 1985. La réparation a consisté à injecter du coulis de ciment à partir d'un forage situé à $40 \mathrm{~cm}$ du pieu.

\subsection{Injections de ciment}

Après carottage et curage de la colonne de sol intérieure au pieu, un bouchon de ciment a été coulé à l'extrémité (fig. 9). Un forage avec un outil de $13,3 \mathrm{~cm}$ de diamètre a été réalisé à $40 \mathrm{~cm}$ du pieu et jusqu'à $22 \mathrm{~m}$ de profondeur. Le contrôle des positions relatives du trou de forage et du pieu a été effectué par inclinométrie.

Un tube à manchettes (TAM) de $7,6 \mathrm{~cm}$ de diamètre, fermé à son extrémité, a été descendu dans le trou de forage puis cimenté. Le TAM est un tube percé tous les $50 \mathrm{~cm}$, les trous étant protégés par des manchettes en caoutchouc (fig. 10). Après prise du ciment, il est possible de descendre dans le TAM un

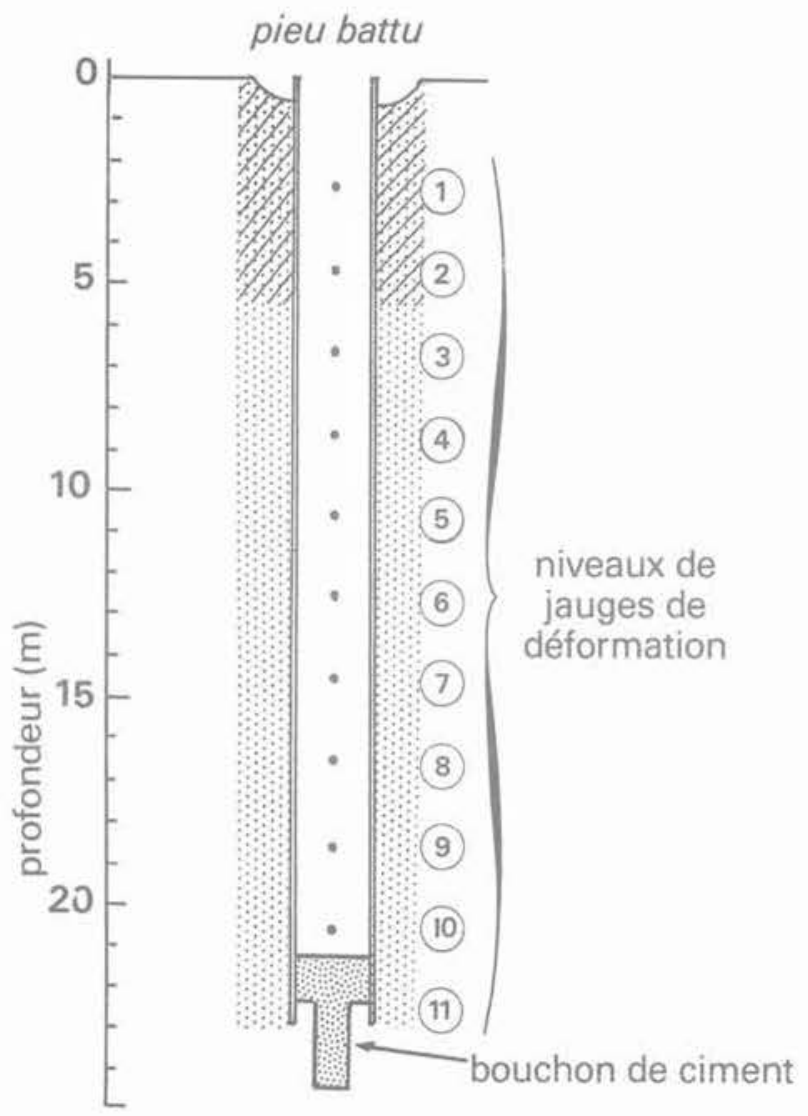

Fig. 9. - Section schématique du pieu A'.

Fig. 9. - Schematic section of the pile A'

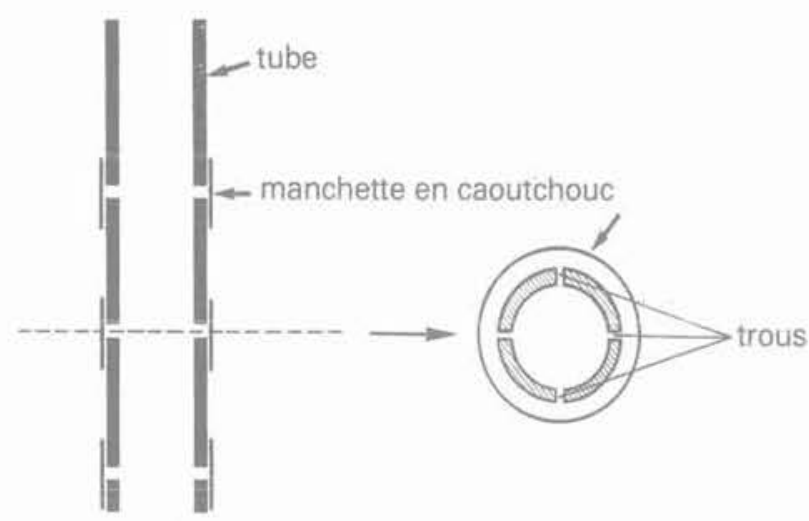

Fig. 10. - Tube à manchette ou TAM. Fig. 10. - Sleeve tube or TAM

double obturateur qui permet d'isoler une manchette et d'injecter de l'eau ou du ciment (NAUROY et al. 1987) (fig. 11),

Deux injections successives de coulis ont été réalisées: - la première de 270 litres de coulis préparé avec un rapport ciment/eau de 2, limitée aux niveaux 17 , 18 et $19 \mathrm{~m}$ (fig. 12);

- la seconde de 1325 litres de coulis, généralisée entre les niveaux 12 et $21 \mathrm{~m}$, la pression étant limitée par l'appareillage à $10 \mathrm{MPa}$. 


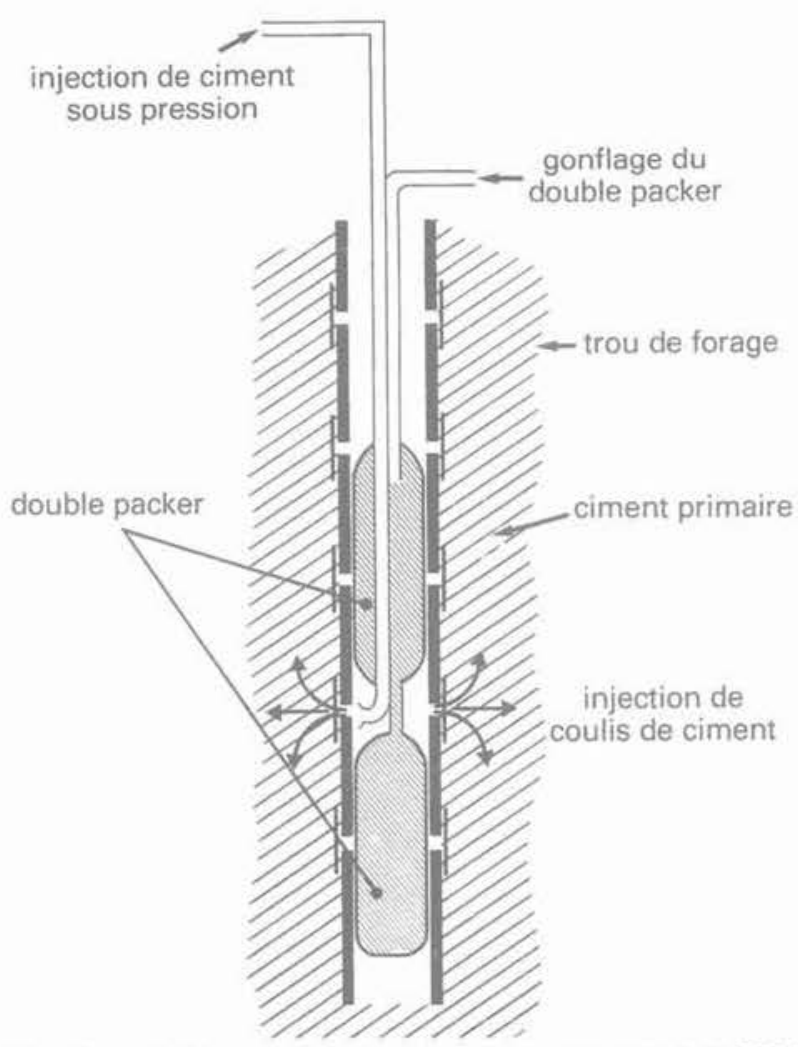

Fig. 11. - Mise en ceuvre du double obturateur dans le TAM pour injection de ciment.

Fig. 11. - Implementation of the double packer in TAM for grout injection.

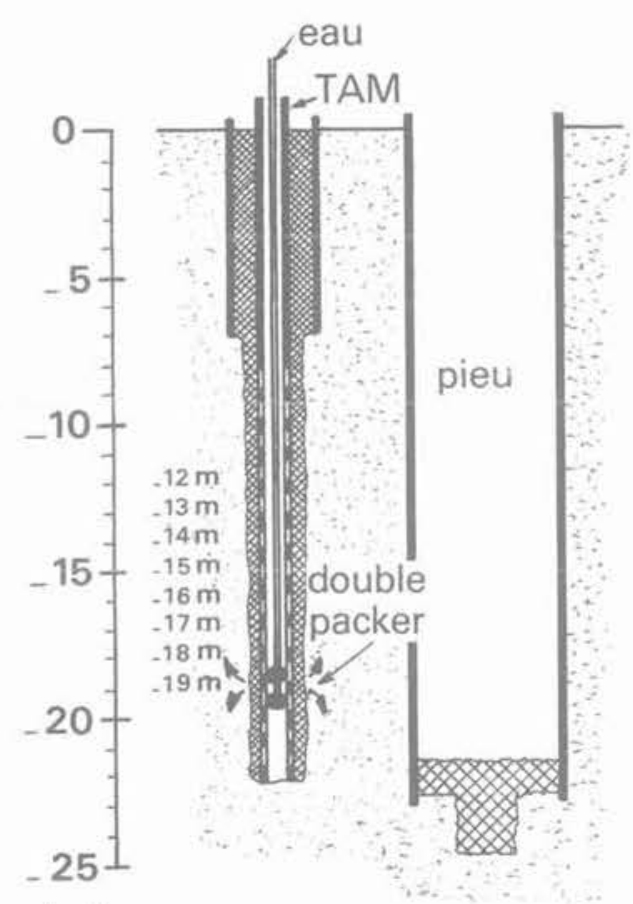

(m)
Des essais de traction ont été réalisés après chaque injection.

\subsection{Essais de traction}

Trois essais de chargement statique en traction ont été effectués:

- T2 après la première injection,

- T3 et T4 après la seconde injection.

L'appareillage et les procédures utilisées pour les essais T2 et T3 étaient semblables à celles décrites précédemment pour le pieu A (cf. chap. 2).

Pour l'essai T4, un collier d'extraction permettant de reprendre une charge de $3000 \mathrm{kN}$ fut utilisé.

Les résultats des essais T2. T3 et T4 sont comparés à ceux de l'essai de chargement statique T1 réalisé sur le pieu A juste après battage (fig. 13). L'influence des injections est manifeste: la capacité portante est multipliée par trois après la première injection et par plus de trente après la seconde.

Au cours de l'essai T4, la capacité limite du pieu n'a pas été atteinte pour $2100 \mathrm{kN}$, charge pour laquelle la contrainte dans l'acier atteint la limite admise de $280 \mathrm{MPa}$.

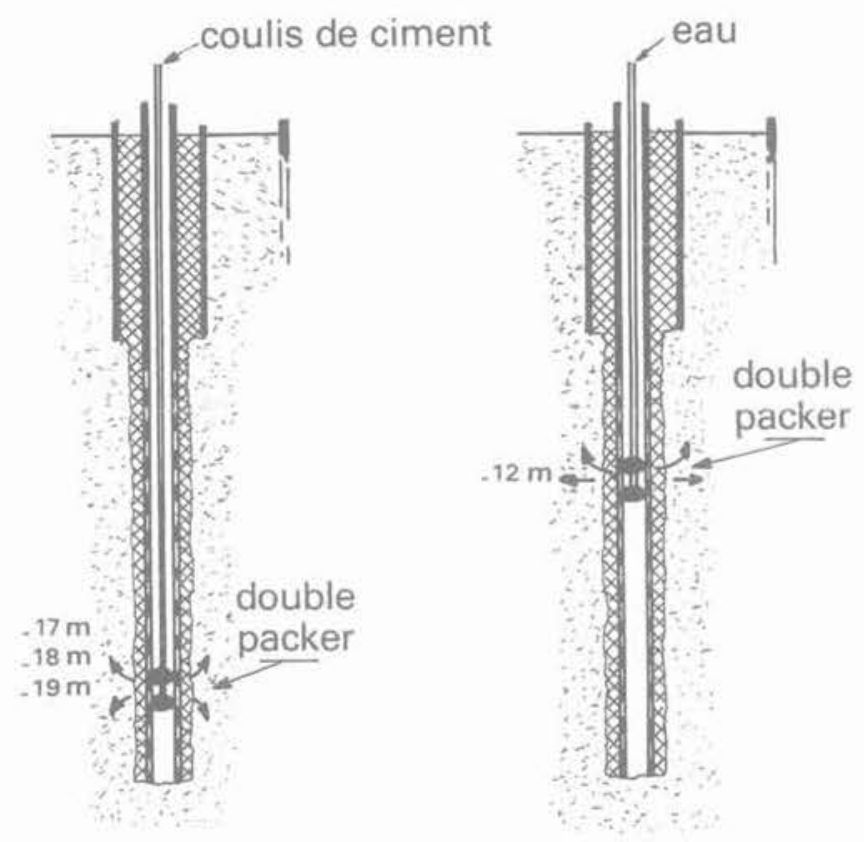

Injection
(c) eau de lavage

Fig. 12. - Procédure pour la première injection de ciment. Fig. 12. - Procedure for the first grout injection. 


\subsection{Répartition du ciment autour du pieu}

Le volume de sol entourant le pieu et le TAM a été découpé en vibrofonçant une palplanche équipée d'un système de lançage. Une traction a ensuite été exercée sur le pieu et le TAM reliés en tête. Le pieu a été extrait du sol en une seule opération.

La gaine de ciment observée est très rugueuse et répartie autour du pieu sur une épaisseur de 5 à $20 \mathrm{~mm}$ environ (fig. $14 \mathrm{~b}$ ). Une excroissance de $10 \mathrm{~cm}$ d'épaisseur, opposée au TAM, est observée à la cote $15 \mathrm{~m}$. Partout le ciment est pur, de densité comprise entre 20 et $21 \mathrm{kN} / \mathrm{m}^{3}$ supérieure à la valeur de $18 \mathrm{kN} / \mathrm{m}^{3}$ initiale, ce qui traduit l'importance de la migration de l'eau dans le terrain. On a également observé des traces de digitations de $1 \mathrm{~mm}$ d'épaisseur radiales au pieu. Ce sont probablement les restes d'un réseau de digitations reliant le TAM et le pieu, brisées lors de l'extraction.

Le volume moyen de la gaine de ciment est inférieur au quart du volume total de ciment injecté; ainsi la majeure partie du ciment est restée dans le sol autour du TAM et dans l'espace compris entre le TAM et le pieu. Il n'est pas possible de distinguer l'impact de chaque injection séparément car le coulis injecté avait la même couleur. On constate simplement que le coulis a migré vers le haut à partir des points d'injection (fig. 14 a et 14 b).

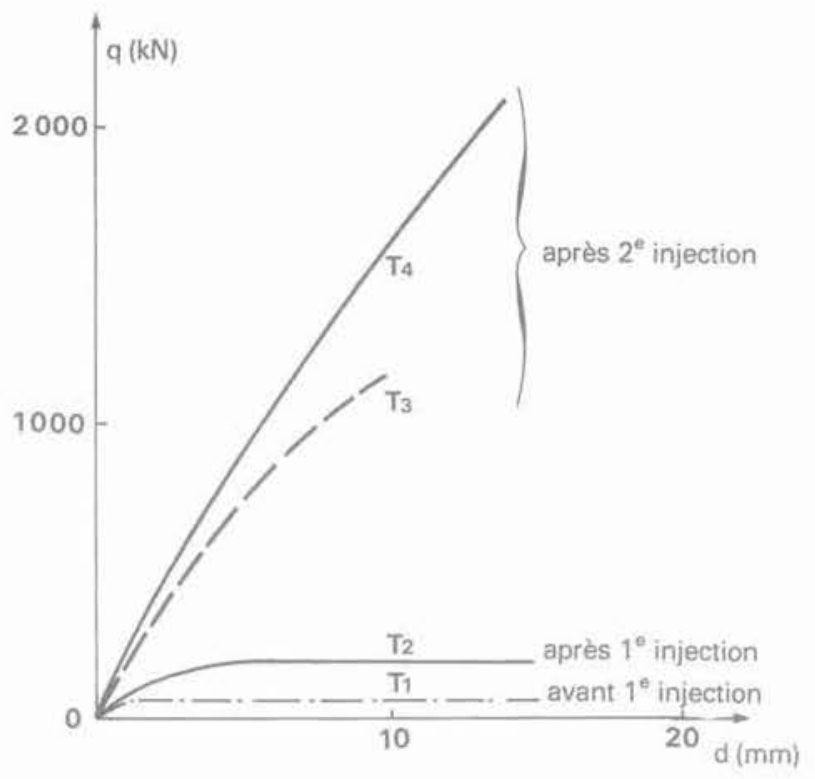

Fig. 13. - Courbes effort-déplacement pour les essais statiques en traction.

Fig. 13. - Load displacement curves for static tension tests.

\subsection{Mobilisation du frottement latéral le long du pieu}

L'enregistrement des élongations des jauges au cours des essais T2 et T3 a permis de déterminer la distribution des charges dans le pieu et le frottement latéral mobilisé. La figure $14 \mathrm{c}$ montre le frottement maximal mobilisé, calculé en tenant compte d'une épaisseur moyenne de la gaine de ciment le long du pieu.

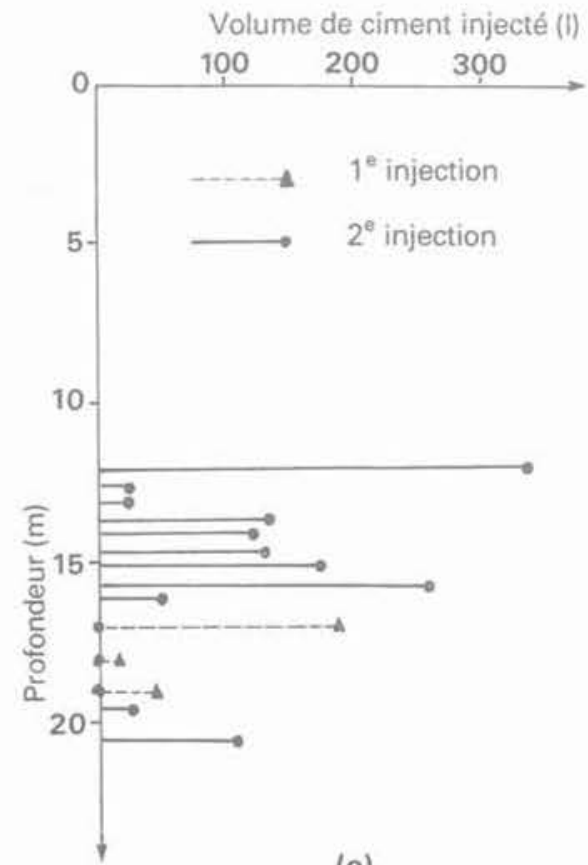

(a)

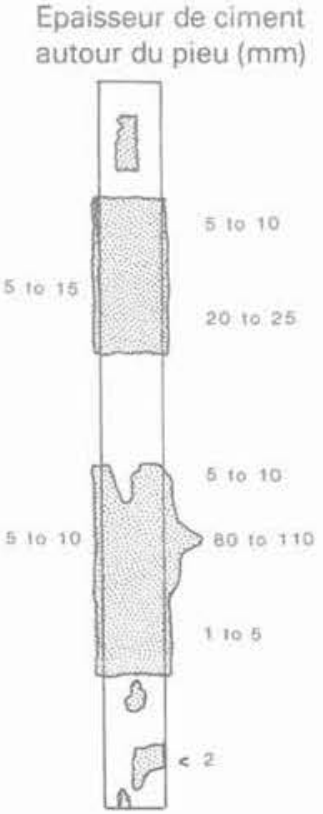

(b)

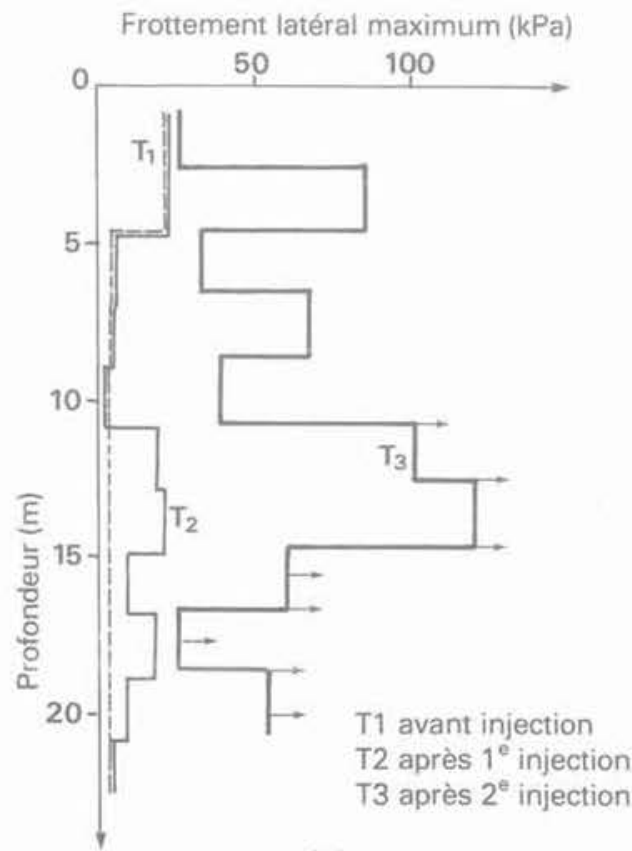

(c)

Fig. 14. - Répartition du ciment autour du pieu $A^{\prime}$ et frottement latéral mobilisé.

Fig. 14. - Distribution of grout around the pile $A^{\prime}$ and skin friction mobilized. 
Après la première injection de ciment (essai T2), le frottement s'est accru entre 11 et $20 \mathrm{~m}$ jusqu'à une valeur assez uniforme de $20 \mathrm{kPa}$. Ceci traduit l'influence d'une injection limitée en volume et localisée sur une hauteur de $2 \mathrm{~m}$ : le coulis de ciment migre pour couvrir une zone plus vaste et excentrée vers le haut. Par ailleurs, l'observation du comportement frottant du pieu pour un déplacement de $12 \mathrm{~cm}$ semble indiquer que les digitations entre le TAM et le pieu ne jouent aucun rôle et qu'il n'y a pas d'effet de butée.

Après la deuxième injection (essai T3), le frottement latéral s'est accru tout le long du pieu, y compris dans le niveau supérieur de sable silteux. Cette observation a confirmé la tendance du coulis à migrer vers le haut lors de linjection.

Entre 0 et $10 \mathrm{~m}$, le frottement latéral est complètement mobilisé et atteint localement $80 \mathrm{kPa}$. Audessous de $10 \mathrm{~m}$, le frottement latéral n'a pu être totalement mobilisé au cours des essais T3 et T4, mais la charge reprise entre 10 et $23 \mathrm{~m}$ est supérieure à $1650 \mathrm{kN}$, ce qui représente un frottement latéral moyen supérieur à $125 \mathrm{kPa}$, valeur voisine de celles observées pour le pieu $\mathrm{B}$ foré et cimenté.

\section{PIEU C BATTU-CIMENTÉ}

Le frottement latéral élevé, mobilisé après injection de ciment autour du pieu $A$, confirme d'une part l'intérêt de la cimentation des pieux dans les sables carbonatés et, d'autre part, les possibilités d'injection de coulis de ciment autour des pieux battus dans les matériaux compressibles. Dans la perspective du développement d'un procédé de pieu battu et cimenté, Solmarine entreprenait, en 1986, sur le même site de Plouasne, une importante expérimentation d'un pieu battu, équipé de lignes de cimentation et clapets pour l'injection de coulis après battage.

\subsection{Description du pieu C}

Le pieu $C$ est un tube ouvert de dimensions:

- diamètre extérieur : $762 \mathrm{~mm}$;

- épaisseur : 19,02 mm;

- longueur : $24 \mathrm{~m}$.

Le pieu est équipé de deux petits tubes de cimentation, soudés par points à l'intérieur du pieu, diamétralement opposés. Huit clapets disposés à $3 \mathrm{~m}$ d'intervalle sont localisés dans les $12 \mathrm{~m}$ inférieurs du pieu. Les lignes de cimentation sont protégées en pied par des sabots (fig. 15).
Le pieu a été instrumenté par des jauges de déformation, collées sur la surface intérieure, selon trois génératrices à $120^{\circ}$ et réparties sur douze niveaux espacés de $1,5 \mathrm{~m}$.

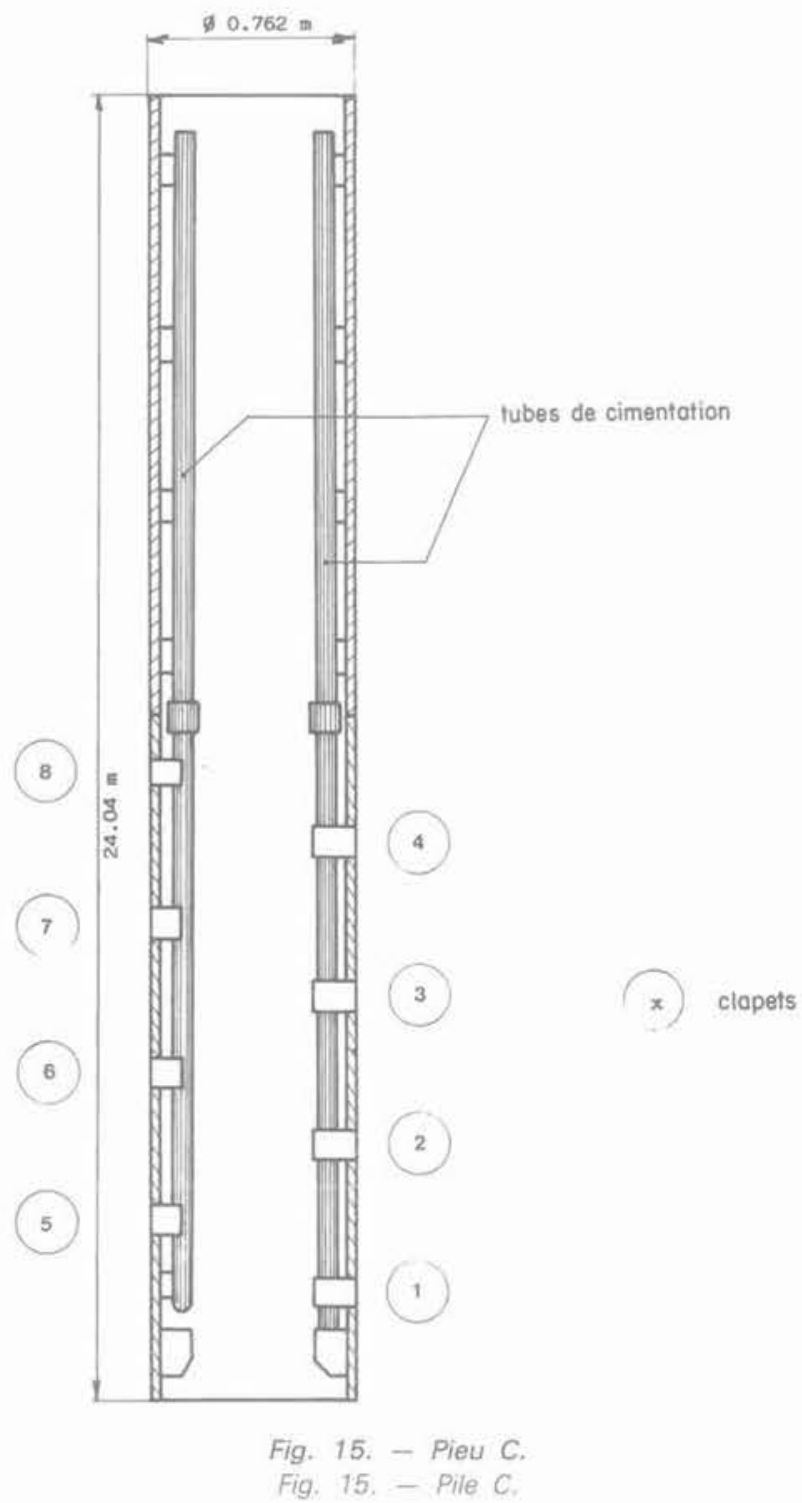

\subsection{Installation du pieu}

A l'emplacement prévu pour le pieu, une excavation fut d'abord creusée pour installer un tube-guide de $1,05 \mathrm{~m}$ de diamètre et $3 \mathrm{~m}$ de longueur. Un second tube-guide, de $91 \mathrm{~cm}$ de diamètre, fut battu à l'intérieur du premier jusqu'à $8,5 \mathrm{~m}$ de profondeur, puis curé par forage.

Le pieu $C$, descendu dans le trou, a été battu avec un marteau Delmag D30 jusqu'à une profondeur de $23,2 \mathrm{~m}$ (fig. 16). 


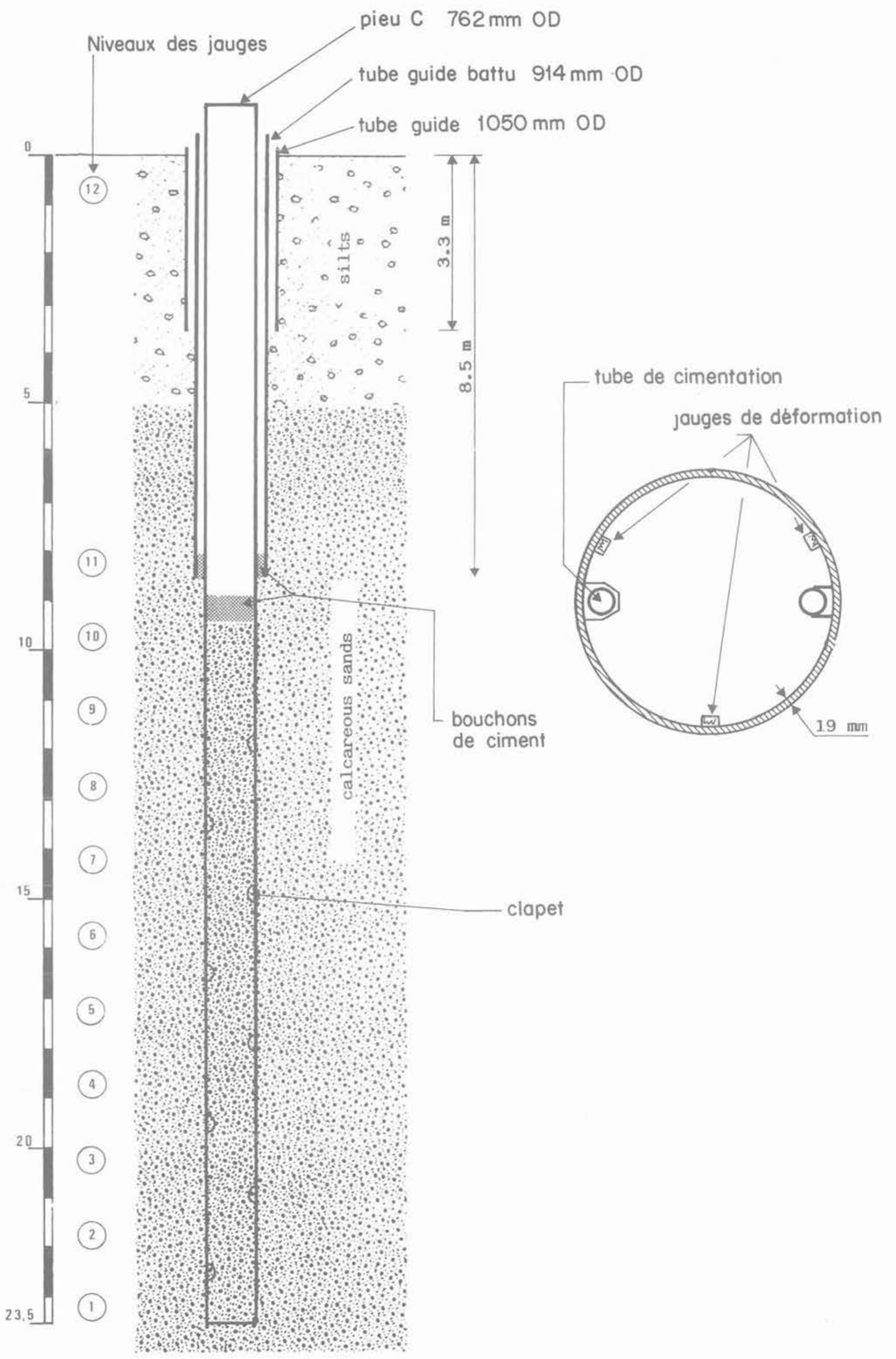

Fig. 16. - Le pieu C battu dans les sables carbonatés de Plouasne. - Fig. 16. - Pile C driven in Plouasne calcareous sands. 


\subsection{Injection de ciment}

Un double obturateur descendu dans une ligne de cimentation permet d'isoler un clapet et d'injecter de l'eau ou du ciment. Deux injections successives de ciment ont été réalisées.

La première a été limitée au clapet 5 situé à $2,55 \mathrm{~m}$ de la pointe du pieu; 70 litres de coulis préparé avec un rapport ciment/eau de 2 ont été injectés.

La seconde injection a été réalisée par les clapets 7 et 8 situés respectivement à $8,55 \mathrm{~m}$ et $11,55 \mathrm{~m}$ de la pointe du pieu; 150 litres de coulis coloré ont été injectés par chaque clapet.

Des essais de traction ont été réalisés après chaque injection.

\subsection{Essais de traction}

Quatre essais de chargement statique en traction ont été réalisés:
- S1 après battage;

- S2 après la première injection:

- S4 et S6 après la deuxième injection.

Pour ces essais, les charges ont été reprises par un bâti de réaction constitué de poutres métalliques reposant sur deux blocs de béton posés de part et d'autre du pieu. La charge était appliquée par un vérin hydraulique de $10 \mathrm{MN}$ lié au pieu par 55 câbles en acier (fig. 17).

La charge était appliquée par incréments maintenus durant une heure.

Les déplacements de la tête du pieu ont été mesurés par un théodolite et un système NIVOMATIC par rapport à une référence située à une dizaine de mètres.

Les différentes mesures de charge en tête, de déplacement et d'élongation des jauges, ont été enregistrées sur une chaîne d'acquisition numérique.

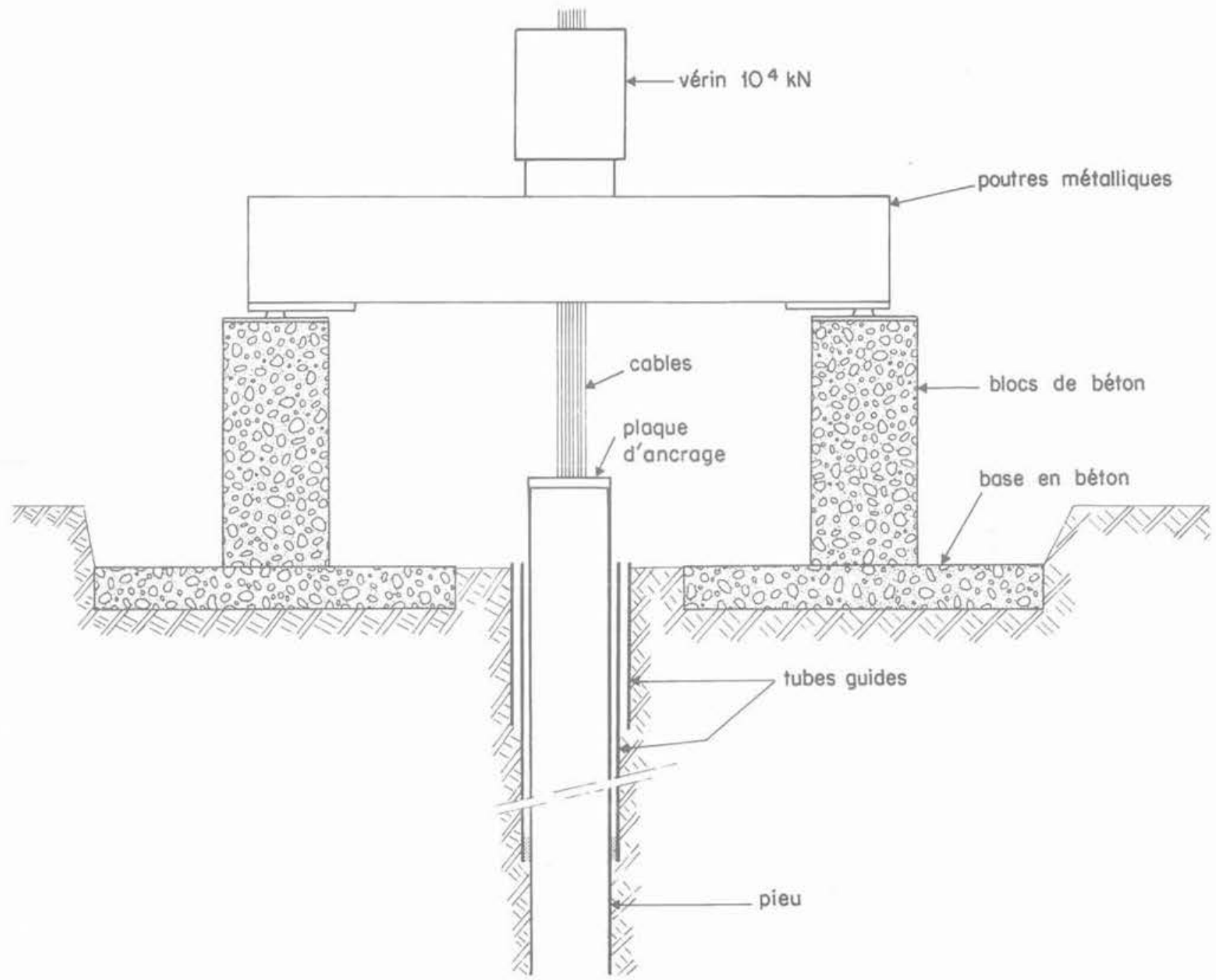

Fig. 17. - Bâti de réaction utilisé pour le pieu C. Fig. 17. - Reaction frame used for pile C. 


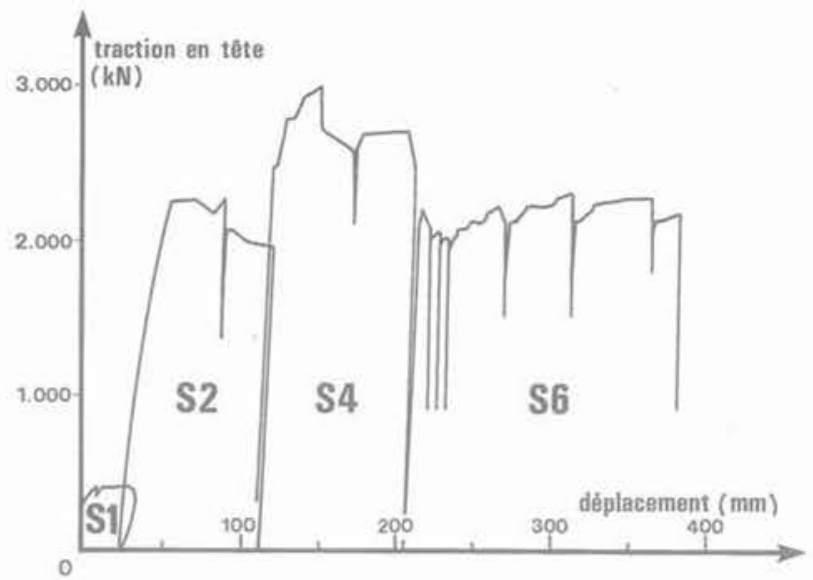

Fig. 18. - Résultats des essais de traction réalisés sur le pieu C. Fig. 18. - Results of tension tests performed on pile $C$.

La figure 18 reproduit les courbes de chargement en tête.

L'essai S1 montre clairement le faible frottement mobilisé par le pieu après battage dans les sables carbonatés et confirme les résultats obtenus avec le pieu A.

La capacité portante a été considérablement accrue par les injections. A l'issue de la seconde injection, la charge de rupture a atteint $3000 \mathrm{kN}$, tandis que

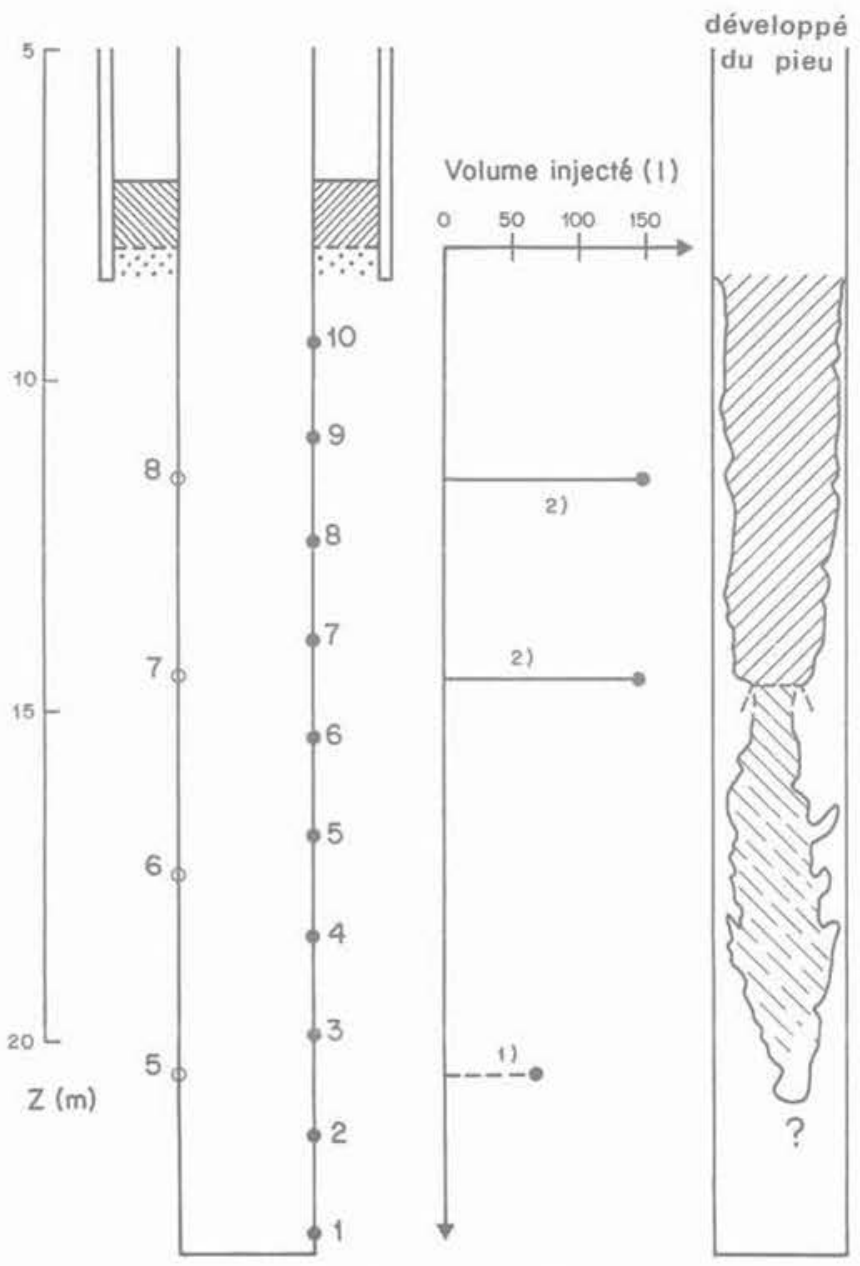

la charge résiduelle s'est stabilisée autour de $2200 \mathrm{kN}$.

\subsection{Répartition du ciment autour du pieu}

Comme pour les autres pieux, le sol a été découpé en vibrofonçant une palplanche équipée d'un système de lançage puis le pieu a été extrait du sol.

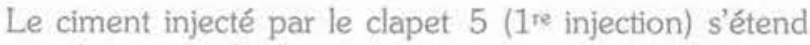
sur $6 \mathrm{~m}$ vers le haut du pieu, avec une épaisseur variant de 4 à $10 \mathrm{~mm}$ et atteignant $40 \mathrm{~mm}$ au droit du clapet. La gaine de ciment centrée sur la génératrice du clapet, couvre seulement $45 \%$ de la surface du pieu, mais quelques fragments ont sûrement été détruits en cours d'extraction. Le ciment injecté par le clapet 7 ( $2^{e}$ injection) est remonté également sur une hauteur de 7 à $8 \mathrm{~m}$ jusqu'au tube-guide (fig. 19). La gaine de ciment couvre à peu près $65 \%$ de la surface avec une épaisseur variable de 5 à $10 \mathrm{~mm}$ du côté clapet, mais plus faible du côté opposé, avec de nombreuses digitations. Le ciment, souvent mêlé au sable carbonaté, n'est pur qu'au niveau du clapet. L'aspect de surface est abrasé. Le ciment injecté par le clapet 8 ( $3^{e}$ injection) n'apparait que par traces éparses de faible épaisseur sous le ciment injecté par le clapet 7.

Fig. 19. - Répartition du ciment autour du pieu $C$ et frottement latéral mobilisé. Fig: 19. - Distribution of grout around the pile $C$ and skin friction mobilized.

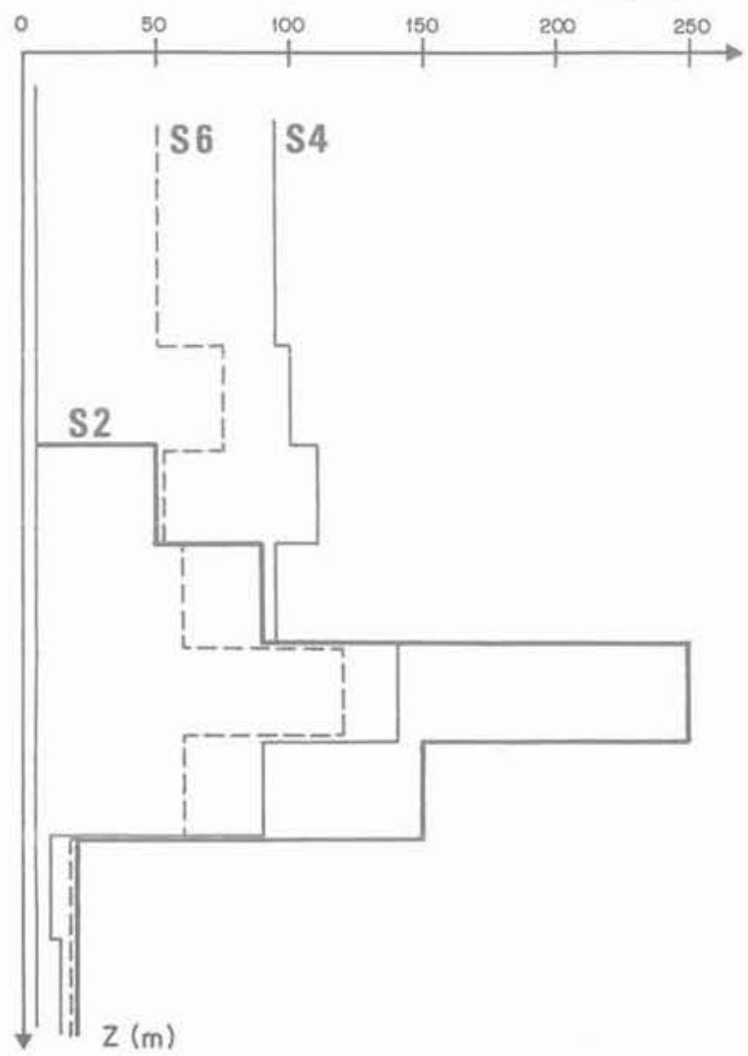




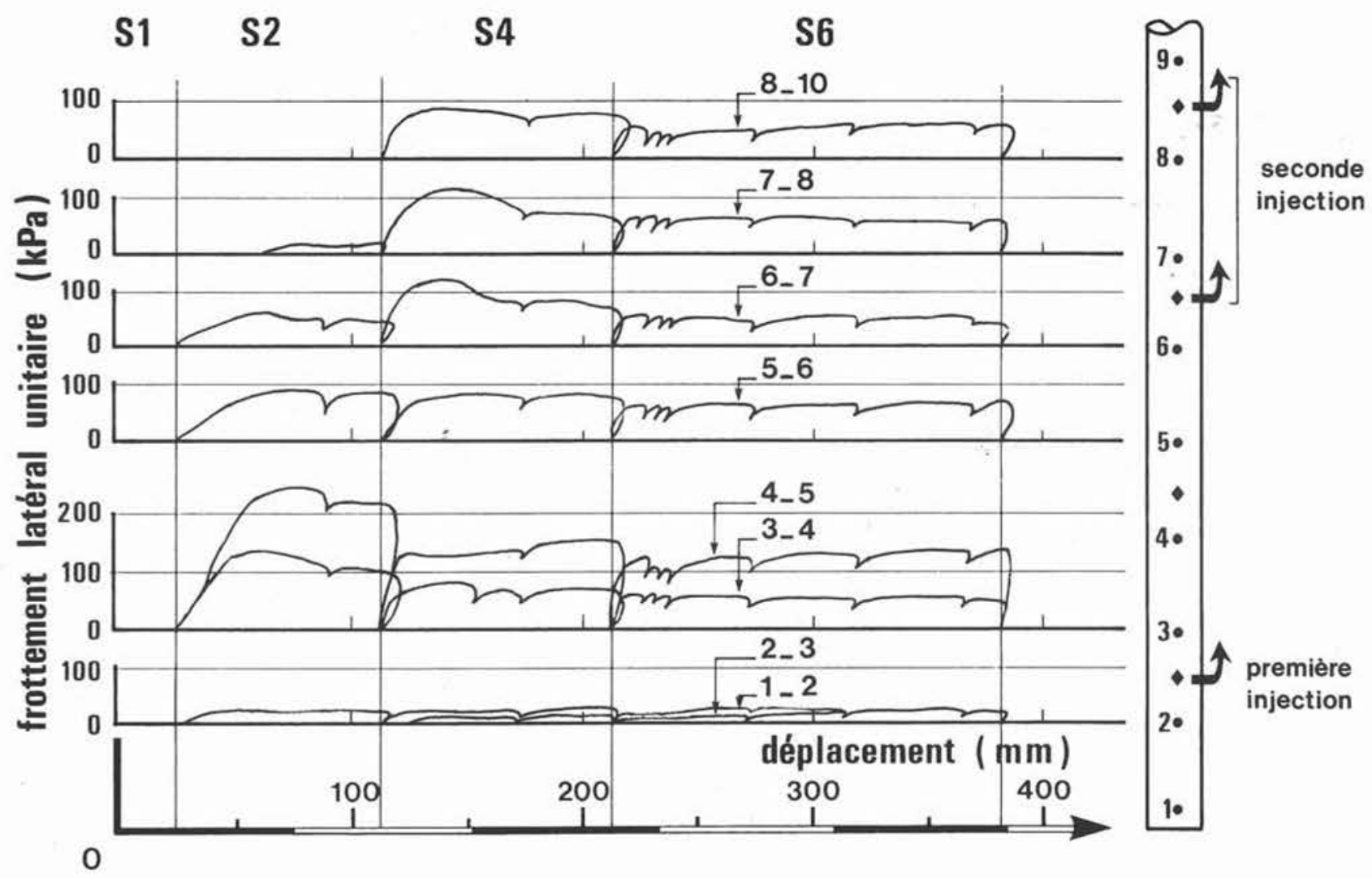

Fig. 20. - Mobilisation du frottement latéral le long du pieu C.

Fig. 20. - Mobilization of skin friction along the pile C.

\subsection{Frottement latéral mobilisé}

La figure 19 montre le frottement maximal mobilisé au cours des essais S1, S2 et S4, ainsi que le frottement résiduel au cours de $\mathrm{S} 6$.

Après battage, le frottement est inférieur à $5 \mathrm{kPa}$ sur toute la longueur du pieu.

Après la première injection par un clapet unique, le frottement s'est accru dans une zone de $6 \mathrm{~m}$ de long située au-dessus du clapet d'injection.

Le frottement est très élevé entre les niveaux 3 et 5 où l'on constate un enrobage important. Le frottement maximal atteint $250 \mathrm{kPa}$, valeur supérieure au frottement mobilisé par le pieu foré et cimenté. Il convient cependant d'être prudent dans toute extrapolation car, entre 17 et $20 \mathrm{~m}$, le pieu $\mathrm{C}$ se trouve dans une zone de résistance plus élevée au pénétromètre.

Après la seconde injection, le frottement s'est amélioré tout le long du pieu jusqu'au tube-guide. On observe une bonne corrélation entre l'enrobage du pieu et le frottement latéral (fig. 19). Si pour les niveaux $3 / 4$ et $5 / 6$ on observe une bonne continuité entre le frottement mobilisé à la fin de l'essai S2 et celui mobilisé au début de l'essai S4, en revanche, on doit constater une perte sensible de frottement au niveau $4 / 5$ par rupture (fig. 20).

Le frottement semble ensuite se stabiliser autour de 50 à $70 \mathrm{kPa}$ le long du pieu (essai S6).

\section{CONCLUSIONS}

Les expérimentations opérées et les résultats obtenus avec différents types de pieux mis en place dans les sables carbonatés compressibles de Plouasne, représentatifs de ceux rencontrés en de nombreuses zones d'activités pétrolières en mer, ont largement contribué à l'amélioration du choix et du dimensionnement des pieux dans les formations carbonatées en mer.

1. Le frottement latéral des pieux battus dans les sables carbonatés de Plouasne ne dépasse pas 3 à $5 \mathrm{kPa}$. Ces résultats sont en bon accord avec les mesures (ou les déductions) effectuées sur des pieux battus dans les sables carbonatés non cimentés sur divers sites de production en mer. 
2. Le frottement latéral des pieux forés et cimentés dans les sables carbonatés dépasse largement $100 \mathrm{kPa}$ mais le frottement résiduel se réduit à environ $50 \mathrm{kPa}$ après un déplacement du pieu équivalent à un demidiamètre. Le choix du procédé de pieux forés et cimentés, pour la réalisation des fondations d'ouvrages en mer dans les formations carbonatées, doit cependant prendre en compte les difficultés de réalisation des forages (risques d'éboulement) et le coût probablement élevé (durée d'immobilisation prolongée des moyens navals et des équipements).

3. La «réparation» des pieux battus par injection de ciment depuis un forage parallèle au pieu est réalisable, le frottement latéral atteignant des valeurs comparables (>100 $\mathrm{kPa}$ ) à celles mobilisées par les pieux forés et cimentés. Les difficultés évidentes de reprise des fondations des ouvrages en mer peuvent conduire cependant à limiter ce genre d'interventions.

4. Le procédé de pieu battu et injecté associe la facilité de mise en place des pieux par battage et la bonne capacité portante en frottement mobilisée par les pieux forés et cimentés. Les résultats du pieu C expérimental de Plouasne, confirmés depuis par d'autres expérimentations en Australie conduites par Solmarine pour le compte d'Esso Australie (COTTRIL, 1988), confirment la fiabilité de ce procédé relativement économique par rapport au procédé de pieux forés et cimentés.

En conclusion, les expérimentations de Plouasne ont ouvert de nombreuses voies de recherche en vue d'améliorer la sécurité des ouvrages de production pétrolière dans plusieurs zones d'activités en mer. Le suivi des expérimentations et des réalisations en cours ou prévues devrait permettre de juger, dans un avenir proche, de l'efficacité technique et économique des travaux réalisés dans ce domaine par l'ARGEMA.

\section{BIBLIOGRAPHIE}

BARTHÉLEMY H., GOUVENOT D., MARTIN R., BUSTAMANTE M. (1986), "Pressure Grouted Piles: A Solution to Offshore Foundation Pro- blems». Proc. 3rd Int. Conf. on Numerical Methods in Offshore Piling, Nantes, Editions Technip.

BARTHÉLEMY H., MARTIN R., LE TIRANT P., NAUROY J.F., CIPRIANO DE MEIDEROS J. (1987), « Grouted Driven Piles: An Economic and Safe Alternate for Pile Foundation». Proc. 19 th Off. Techn. Conf. Houston, paper OTC 5409.

BRUCY F., MEUNIER J., NAUROY J.F. (1988), "Analysis of pile driving tests in calcareous sand sites ». Proc. of 3rd Int. Conf. on the Applications of Stress-Wave Theory on Piles». Ottawa.

COTTRIL A. (1988), "Offshore Australia». Offshore Engineer, october 1988.

JEFFRIAUD J.P., BARTHÉLEMY H. (1984), «Dispositif comportant une valve pour l'injection de coulis d'un pieu tubulaire enfoncé dans le sol». Brevet Solétanche $n^{\circ} 84-13500$ déposé le 31.08.84.

NAUROY J.F., LE TIRANT P. (1983), «Models Tests of Piles in Calcareous Sands». Proc. ASCE Conf. on Geotechnical Practice in Offshore Engineering, University of Texas, Austin.

NAUROY J.F., LE TIRANT P. (1985), «Static and Cyclic Load Tests on a Drilled and Grouted pile in Calcareous Sand». Proc. Int. Conf. on Behavior of Offshore Structures, BOSS". 85, Delft.

NAUROY J.F., BRUCY F., LE TIRANT P., KERVADEC J.P. (1986), "Design and Installation of Piles in Calcareous Formations». Proc. 3rd Int. Conf. on Numerical Methods in Offshore Piling, Nantes, Editions Technip.

NAUROY J.F., BRUCY F., LE TIRANT P., BARTHÉLEMY' H., KERVADEC J.P. (1987), «Repair of Driven Piles in Calcareous Formations». Proc. of Int. Symp. on Offshore Engineering, Brasil Offshore'87, Pentech Press.

NAUROY J.F., BRUCY F., LE TIRANT P. (1988), "Skin Friction of Piles in Calcareous Sands". Proc. of Int. Conf. on Calcareous Sediments, Perth, R.J. Jewell, D.C. Andrews eds., A.A. Balkema, Rotterdam, 1988. 